\title{
Risks and burdens of third-generation cephalosporin resistant Enterobacteriaceae in neonatal sepsis
}

\author{
Thatrimontrichai, A. ${ }^{*}$, Premprat, N. ${ }^{1}$, Janjindamai, W. ${ }^{1}$, Dissaneevate, S. ${ }^{1}$ and Maneenil, G. ${ }^{1}$ \\ ${ }^{1}$ Division of Neonatology, Department of Pediatrics, Faculty of Medicine, Prince of Songkla University, \\ Songkhla, Thailand \\ *Corresponding author e-mail: tanucha@medicine.psu.ac.th \\ Received 1 April 2020; received in revised form 6 July 2020; accepted 7 July 2020
}

\begin{abstract}
Third-generation cephalosporin resistant Enterobacteriaceae (TCRE) is a global concern especially in neonatal sepsis. We performed a secondary data analysis in a Thai neonatal intensive care unit to identify the risk factors for acquisition of TCRE sepsis and mortality of Enterobacteriaceae sepsis between 1991 and 2017. Multivariate logistic and Cox proportional regression were used for analysis. Numbers of neonates with TCRE and nonTCRE sepsis were 100 and 41 patients, respectively. Medians (interquartile ranges) of gestational age, birthweight, onset of sepsis and total hospital stay of neonates with Enterobacteriaceae sepsis were $32(28,38)$ weeks, $1670(1025,2750)$ grams, $11(6,25)$ days and $41(22,74)$ days, respectively. Univariate and multivariate analysis, neonates with TCRE sepsis were more likely to have birth asphyxia (adjusted odds ratio $[\mathrm{aOR}]=2.6$; 95\% confidence interval [CI] 1.1-6.0; $p=0.02$ ) and history of aminoglycoside exposure (aOR $=2.9 ; 95 \%$ CI 1.3$6.7 ; p=0.01)$. In-hospital case fatality rate from Enterobacteriaceae sepsis was $26 \%(36 / 141)$. In Cox regression, neonates with TCRE sepsis was not an independent risk of non-survivors, but septic shock (adjusted hazard ratio $=9.9 ; 95 \%$ CI 5.0-19.7, $p<0.001$ ) increased 30-day mortality in the final model. Asphyxia and previous aminoglycoside consumption were risks of acquisition for neonatal TCRE sepsis while the burden was not a significant difference. Infection prevention and control must be strictly implemented in high multidrug-resistant area.
\end{abstract}

\section{INTRODUCTION}

Neonatal sepsis is a worrisome concern especially multidrug-resistant pathogen. Trends of late onset Gram negative bacilli (GNB) bacteremia, especially due to the Enterobacteriaceae family, increased in a neonatal intensive care unit (NICU) (Thatrimontrichai et al., 2014) and was associated with a higher case fatality rate (CFR) (Cohen-Wolkowiez et al., 2009). Emergence of extended-spectrum betalactamase (ESBL) producing or thirdgeneration cephalosporin resistant Enterobacteriaceae (TCRE) in neonatal sepsis is a concern due to the consideration of off-label use of meropenem and colistin in neonates (Simon \& Tenenbaum, 2013; Thatrimontrichai et al., 2013; Thatrimontrichai et al., 2016).
However, antimicrobial-resistant bacteria may colonize from antibiotic selective pressure due to frequent and prolonged broad spectrum antimicrobial use. The emergence of multidrug-resistant GNB sepsis is particularly worrisome due to growing off-label use of antibiotics such as meropenem and colistin (Thatrimontrichai et al., 2013). On the other hand, there are higher CFR in neonates who receive inadequate empiric antimicrobial therapy in high multidrug-resistant areas (Tsai $e t$ al., 2014).

There are few reports on neonatal TCRE sepsis. Therefore, we aimed to clarify the risk factors for TCRE sepsis and mortality of neonatal Enterobacteriaceae sepsis in Thailand. 


\section{MATERIALS AND METHODS}

\section{Settings and Study Design}

This study was conducted at the NICU of Songklanagarind Hospital, Songkhla, Thailand. The NICU is a 15-bed, multi-bed ward in a university-affiliated teaching hospital at Prince of Songkla University. There are approximately 2500-3500 live births with about 400-550 neonates admitted annually to the NICU.

This is a secondary analysis and retrospective study. Study subjects were identified from the hospital clinical microbiology laboratory database. All records were also reviewed to ensure that all eligible subjects were identified. Medical records of all neonates with Enterobacteriaceae sepsis obtained at any time during admission to the NICU from January 1, 1991 to December 31, 2017 were reviewed. TCRE and non-TCRE groups were neonates who had Enterobacteriaceae organisms from the cultures of blood or cerebrospinal fluid (CSF) or both. We performed surveillance for multidrug resistant (MDR) rates with attempts to identify any potential point source outbreak, if the MDR rates have been increased significantly. However, there was no evidence of outbreak during the entire study period.

Medical records from the medical record unit at Songklanagarind Hospital were reviewed. Recorded data of the patients included obstetric, intrapartum, and neonatal data such as patient demographic factors, devices used (i.e., ventilator or central line [CL]), antimicrobial use, antimicrobial susceptibility, and outcomes.

\section{Definitions}

Onset of sepsis was defined as the postnatal age developed signs of neonatal bacteremia or meningitis and obtained the culture. Birth asphyxia was defined as Apgar score less than 7 at 5 minutes of life. Neurologic disease was defined meningoencephalocele, spina bifida or surgical neurological sequelae. Cardiovascular disease was defined as congenital heart disease that is composed of acyanotic heart disease with signs of congestive heart failure and cyanotic heart disease. Gastrointestinal disease included esophageal atresia, pyloric stenosis, small or large bowel obstruction, omphalocele and gastroschisis.

Previous antimicrobial (third generation cephalosporins [cefotaxime or ceftazidime], cefoperazone-sulbactam, carbapenems [imipenem or meropenem] and aminoglycosides [gentamicin or amikacin]) exposure was defined as intravenous antibiotic use for at least 72 hours before obtaining the culture. CL was defined as an umbilical arterial/venous catheter or catheter by the cutdown technique. Device use and total parenteral nutrition were considered as risk factors if they occurred in the 7 days preceding the onset of sepsis. Criteria for the diagnosis of ventilatorassociated pneumonia (VAP) and CLassociated bloodstream infection (CLABSI) followed the Centers for Disease Control and Prevention and National Healthcare Safety Network guidelines for infants $<1$ year old (Thatrimontrichai et al., 2019a; Thatrimontrichai et al., 2019b).

Inadequate empirical antimicrobial therapy (IEAT) was defined as the use of antimicrobials more than 48 hours after the day that either blood or CSF culture were performed that did not cover the pathogens causing sepsis or administration of antimicrobials that failed to cover the resistant pathogens (Thatrimontrichai et al., 2013). Patients were defined as having septic shock if they had sepsis plus hypotension (gestational age-dependent), persistent tachycardia or reduced peripheral perfusion evidence and needed vasopressor agents to maintain blood pressure within 48 hours after the onset of sepsis (Goldstein et al., 2005). The 3-day, 7-day, 30-day, and inhospital CFRs were calculated by dividing the number of deaths from sepsis by the number of individuals diagnosed with the sepsis on 3, 7 and 30 days after the onset of sepsis, and at discharge, respectively.

\section{Microbiological Methods}

All Enterobacteriaceae isolates were identified to the species level by standard laboratory methods. The isolates were cultured from blood and CSF. For blood 
culture, a minimum of $1 \mathrm{~mL}$ of blood was incubated for five days at $35.5^{\circ}$ or until there was a positive signal in the blood culture systems (BACTEC FX ${ }^{\mathrm{TM}}$ ). Positive culture was routinely performed using a blood agar plate (BAP), MacConkey agar, and chocolate agar. Non-centrifuged CSF was inoculated into Thioglycolate broth and cultured on BAP, MacConkey agar, and chocolate agar. The culture plates and broth were then incubated at $35^{\circ} \mathrm{C}$ with or without $5 \%$ carbon dioxide and examined daily for 2 and 10 days, respectively. The isolated organisms were identified by biochemical tests and the MLab system. Susceptibility testing was performed by Kirby-Bauer disc diffusion method on Muller-Hinton agar plates and interpreted according to Clinical and Laboratory Standards Institute (CLSI) guidelines of the year of isolation (CLSI, 2018).

A TCRE organism was defined as Enterobacteriaceae that was non-susceptible to ceftriaxone, cefotaxime, ceftazidime and aztreonam but susceptible to tazobactam, cefoxitin, sulbactam and carbapenems, then others were incompatible with the above definition as non-TCRE organisms.

\section{Statistical Analysis}

$R$ program was used to develop a database of categorical and continuous variables. Categorical variables are presented as frequency and percentage and were compared using the $\chi^{2}$ test or Fisher's exact test. Non-parametric continuous variables were presented as median (interquartile range [IQR]) and compared using the MannWhitney U-test. All $p$ values were two-tailed and $P$ values less than 0.05 indicated statistical significance. We performed survival analysis by time-to-event (death within 30 days) after the onset of sepsis between TCRE and non-TCRE organisms.

Independent variables with $p<0.2$ in the univariate analysis were retained for a regression model. The model with the lowest Akaike information criteria was judged as the most parsimonious model. Adjusted odds ratio (aOR) and hazard ratio (HR) with $95 \%$ confidence intervals (CI) were computed for variables independently associated with TCRE and non-TCRE groups by multivariate logistic regression model and nonsurvivors and survivors in Enterobacteriaceae sepsis by Cox proportional hazards model, respectively.

\section{RESULTS}

Between 1991 and 2017, the number of neonates with GNB and Enterobacteriaceae sepsis were 275 and 141 cases, respectively. The percentage of TCRE from Enterobacteriaceae sepsis was $71 \%(100 / 141)$. The percentages (neonates) of GNB, Enterobacteriaceae and TCRE sepsis in 10,341 neonates admitted to the NICU for the 27 years were $2.7 \%$ (275), 1.4\% (141) and 1.0\% (100), respectively. The prevalences (neonates) of GNB, Enterobacteriaceae and TCRE sepsis in 78,248 inborn neonates were 1.9 (152), 1.0 (79) and 0.7 (57) per 1,000 live births, respectively. Medians (IQRs) of gestational age, birth weight, onset of sepsis and total hospital stay of neonates with Enterobacteriaceae sepsis were $32(28,38)$ weeks, $1670(1025,2750)$ grams, $11(6,25)$ days and $41(22,74)$ days, respectively. There was no significant difference in onset of sepsis between TCRE and non-TCRE groups $(p>0.05)$. The number (percentage) of TCRE bacteremia was 97 (97) higher than 36 (88) non-TCRE bacteremia $(p<0.05)$. In-hospital CFRs from Enterobacteriaceae sepsis was $26 \%(36 / 141)$.

The results of univariate (Table 1) and multivariate (Table 2) analyses of the risk factors for acquisition in TCRE group was more likely to have birth asphyxia (aOR = 2.6; 95\% CI 1.1-6.0) and exposure to aminoglycosides $(\mathrm{aOR}=2.9 ; 95 \%$ CI 1.3-6.7) than non-TCRE group. Burden of neonatal TCRE sepsis compared with non-TCRE sepsis (excess CFR and duration during admission until discharge were $8 \%$ and 11 days) were not significantly different $(p>0.05$ in Table 1).

By survival analysis, there was not statistically significantly difference in nonsurvivors between the TCRE and nonTCRE sepsis $(\mathrm{HR}=1.6 ; 95 \%$ CI 0.7-3.8; $p=$ 
Table 1. Risk factors and burdens in neonates with third-generation cephalosporin resistant Enterobacteriaceae (TCRE) and non-TCRE sepsis

\begin{tabular}{|c|c|c|c|}
\hline Risk factors & $\begin{array}{l}\text { TCRE group } \\
(\mathrm{n}=100)\end{array}$ & $\begin{array}{l}\text { Non-TCRE group } \\
\quad(\mathrm{n}=41)\end{array}$ & $p$-value \\
\hline GA (week), median (IQR) & $32(28,37)$ & $33(30,38)$ & 0.25 \\
\hline BW (g), median (IQR) & $1695(1000,2728)$ & $1620(1250,2900)$ & 0.30 \\
\hline BW compared to GA & & & 0.42 \\
\hline Appropriate for GA & $75(75)$ & $33(81)$ & \\
\hline Small for GA & $24(24)$ & 7 (17) & \\
\hline Large for GA & $1(1)$ & $1(2)$ & \\
\hline Inborn & $57(57)$ & $22(54)$ & 0.38 \\
\hline Vaginal delivery & $46(46)$ & $22(54)$ & 0.52 \\
\hline Male & $59(59)$ & $23(56)$ & 0.90 \\
\hline Birth asphyxia & $52(52)$ & $11(27)$ & $<0.001$ \\
\hline Onset of sepsis (day), median (IQR) & $14.2(6.4,26.2)$ & $9.1(3.5,18.9)$ & 0.07 \\
\hline \multicolumn{4}{|l|}{ Underlying disease before sepsis } \\
\hline Neurologic disease & $10(10)$ & $3(7)$ & 0.76 \\
\hline Cardiovascular disease & $37(37)$ & $9(22)$ & 0.13 \\
\hline Gastrointestinal disease & $14(14)$ & $9(22)$ & 0.36 \\
\hline Pulmonary hypertension & $5(5)$ & $2(5)$ & 1.00 \\
\hline \multicolumn{4}{|l|}{ Previous antimicrobial exposure } \\
\hline Third-generation cephalosporin & $41(41)$ & $13(32)$ & 0.40 \\
\hline Cefoperazone-sulbactam & $13(13)$ & $1(2)$ & 0.07 \\
\hline Carbapenems & $15(15)$ & $4(10)$ & 0.58 \\
\hline Aminoglycosides & $80(80)$ & $23(56)$ & 0.007 \\
\hline Ventilator use & $64(64)$ & $22(54)$ & 0.34 \\
\hline Ventilator-associated pneumonia & $7(7)$ & $1(2.4)$ & 0.44 \\
\hline CL use & $41(41)$ & $11(27)$ & 0.16 \\
\hline CL-associated bloodstream infection & $7(7)$ & $2(5)$ & 1.00 \\
\hline Total parenteral nutrition & $55(55)$ & $21(51)$ & 0.82 \\
\hline Pathogenic organisms & & & 0.003 \\
\hline Klebsiella spp. & $64(64)$ & $16(39)$ & \\
\hline Escherichia coli & $18(18)$ & $18(43.9)$ & \\
\hline Enterobacter spp. & $18(18)$ & $6(14.6)$ & \\
\hline Serratia rubidaea & $0(0)$ & $1(2.4)$ & \\
\hline Burdens & $\begin{array}{l}\text { TCRE group } \\
(\mathrm{n}=100)\end{array}$ & $\begin{array}{l}\text { Non-TCRE group } \\
(\mathrm{n}=41)\end{array}$ & $p$-value \\
\hline In-hospital CFR & $28(28)$ & $8(20)$ & 0.40 \\
\hline 3-day CFR & $15(15)$ & $4(10)$ & 0.58 \\
\hline 7-day CFR & $18(18)$ & $4(10)$ & 0.33 \\
\hline 30-day CFR & $27(27)$ & 7 (17) & 0.30 \\
\hline \multicolumn{4}{|l|}{ Length of stay (day), median (IQR) } \\
\hline Admission until discharge & $51(26,83)$ & $40(22,63)$ & 0.33 \\
\hline Onset of sepsis until discharge & $23(11,55)$ & $23(13,46)$ & 0.90 \\
\hline
\end{tabular}

Data are presented as n (\%) unless indicated otherwise.

BW indicates birth weight; CFR, case fatality rate; CL, central line; GA, gestational age.

Table 2. Univariate and multivariate analysis in neonates with third-generation cephalosporin resistant Enterobacteriaceae (TCRE) and non-TCRE sepsis

\begin{tabular}{lccc}
\hline Risk factors & $\begin{array}{c}\text { Crude odds ratios } \\
\text { (95\% confidence } \\
\text { interval) }\end{array}$ & $\begin{array}{c}\text { Adjusted odds ratios } \\
\text { (95\% confidence } \\
\text { interval) }\end{array}$ & $\begin{array}{c}p \text {-value } \\
\text { (Wald test) }\end{array}$ \\
\hline Birth asphyxia & $3.0(1.3,6.5)$ & $2.6(1.1,6.0)$ & 0.02 \\
Onset of sepsis & $1.01(0.99,1.03)$ & $0.99(0.98,1.02)$ & 0.85 \\
Cardiovascular disease & $2.1(0.9,4.9)$ & $1.4(0.5,3.5)$ & 0.53 \\
Previous cefoperazone-sulbactam use & $6.0(0.8,47.3)$ & $3.83(0.4,33.4)$ & 0.22 \\
Previous aminoglycosides use & $3.1(1.4,6.9)$ & $2.9(1.3,6.7)$ & 0.01 \\
Central line use & $1.9(0.9,4.2)$ & $1.7(0.7,4.0)$ & 0.25 \\
\hline
\end{tabular}




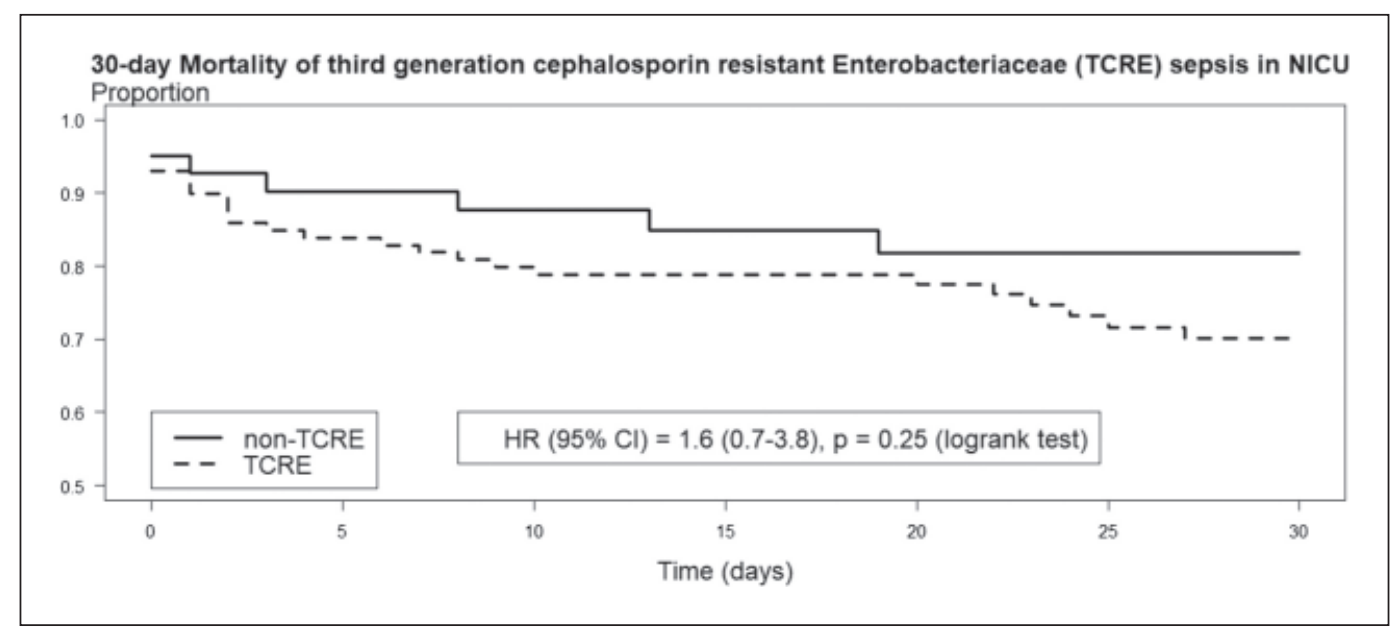

Figure 1. Thirty-day survival rate of neonates with third-generation cephalosporin resistant Enterobacteriaceae (TCRE) and non-TCRE sepsis.

0.25 by logrank test) in Fig. 1. By univariate analysis, risk factors of mortality (nonsurvivors) of Enterobacteriaceae sepsis were composed of weight at onset of sepsis, postmenstrual age, onset of sepsis more than 3 days, birth asphyxia, female and septic shock $(p<0.05)$ when compared with survivors. These variables and TRCE sepsis were put in the regression model then female and septic shock were retained in the final model. In Cox proportional hazards model, septic shock is an independent risk factor of nonsurvivors rather than survivors (adjusted HR $=9.9 ; 95 \%$ CI 5.0-19.7, $p<0.001$ ), whereas for other risk factors including female and TCRE sepsis, there was no significant difference between nonsurvivors and survivors.

\section{DISCUSSION}

Birth asphyxia and previous aminoglycoside exposure of the neonate were associated with TCRE sepsis. However, TCRE sepsis was not a risk factor for mortality but septic shock was a key fatal factor in neonatal Enterobacteriaceae sepsis. Therefore, suspected neonatal sepsis with low Apgar score, previous aminoglycoside exposure and septic shock needs broad-spectrum empirical antimicrobial therapy until the second successive negative culture, especially in a high MDR area (Thatrimontrichai et al., 2013; Thatrimontrichai et al., 2016).

The prevalence of neonatal TCRE/ Enterobacteriaceae sepsis (71\%, 100/141) was similar to Senegal's study (79.7\%, 55/69 neonates) (Breurec et al., 2016). Neonates with risk factors for acquisition in TCRE group were more likely to have birth asphyxia and exposure to aminoglycosides than nonTCRE group. Whereas, the risk factor for infection and/or colonization with ESBLproducing bacteria in the NICU from metaanalysis showed birthweight, gestational age, Caesarean delivery, parenteral nutrition, length of stay in the NICU, mechanical ventilation, central venous catheter use, continuous positive airway pressure, endotracheal intubation, malformations, and previous antibiotic use (ampicillin/ gentamicin, and cephalosporins) (Li et al., 2017). Birth asphyxia may compromise immune defense then increase risk of neonatal infection and previous aminoglycoside consumption may be associated with MDR infection. Previous cephalosporin exposure was not a risk factor of neonatal TCRE sepsis, similar to pediatric ESBLproducing Enterobacteriaceae (ESBL-E) sepsis compared with non-ESBL-E sepsis in Thailand (Nivesvivat et al., 2018). 
The burden (CFR and length of hospital stay) in neonatal and pediatric drug resistant GNB sepsis is still unclear. The in-hospital CFR of Enterobacteriaceae sepsis in our study (25.5\% [36/141]) was higher than in Taiwan (13.9\% [50/360]) (Tsai et al., 2016). The CFR in neonatal MDR GNB sepsis was higher than non-MDR GNB sepsis in Taiwan (Tsai et al., 2014) (28.6\% versus $10.5 \%$, excess $=18.1 \%, p<0.05)$ and Thailand (Thatrimontrichai et al., 2019c) (37.6\% versus $26.1 \%$, excess $=11.5 \%$ ). The CFR in ESBL-E sepsis was higher than non-ESBL-E sepsis in both Taiwan's neonates (Tsai et al., 2016) (19.7\% versus $12.5 \%$, excess $=7.2 \%, p>0.05)$ and Thailand's children (Nivesvivat et al., 2018) (38.9\% versus $13.3 \%$, excess $=25.6 \%$, $p<0.05)$. Excess CFR in our study was $8 \%$ and not significant between two groups ( $p>$ $0.05)$. While the length of hospitalization in neonatal MDR GNB (78.5 versus 75 days)(Tsai et al., 2014), ESBL-E (71 versus 64 days) (Tsai et al., 2016), and TCRE (51 versus 40 days) sepsis were not significantly different compared with non-resistant sepsis.

Risk factors for in-hospital mortality of neonatal ESBL-E sepsis were lower gestational age, birth weight and Apgar score, female, and several underlying chronic conditions (Tsai et al., 2016) in the univariate analysis; similarly, our study showed lower weight at onset of sepsis, postmenstrual age and birth asphyxia, late onset of sepsis, female, and septic shock in TCRE group were higher than in non-TCRE group. In multivariate analysis, risk factors for inhospital mortality of neonatal ESBL-E sepsis were small baby (lower gestational age and birth weight), secondary pulmonary hypertension and complications after bacteremia (Tsai et al., 2016), whereas our study showed only septic shock increased risks for in-hospital mortality of neonatal TCRE sepsis.

This study has some limitations. First, the high prevalence of TCRE sepsis may result from referral bias as the referral center had a high volume of sick neonates and limited resources for infection prevention and control. Second, this study is a retrospective study so susceptibility test according to the most recently and annually edited guidelines of the CLSI were different in disc's zone for the long-time change. The susceptibility test was interpreted by E test and not performed by enzyme producer or serotype of pathogen. Therefore, TCRE sepsis, not ESBL-E sepsis, was defined in this study. Third, we lacked some data for risk factors (such as severity of illness at birth, maternal and neonatal colonization, and rate of antibiotic use in the unit) and sequalae (such as infectious and long-term complications). Final, there was not a significant difference of in-hospital CFR between TCRE and non-TCRE groups due to low power (15\%) from post hoc analysis.

\section{CONCLUSION}

Neonatal risks of acquisition for TCRE sepsis were birth asphyxia and aminoglycoside consumption. Risk of mortality of neonatal Enterobacteriaceae sepsis was septic shock. Active surveillance culture program in asymptomatic neonates should be considered to inform the antibiotic stewardship program, and infection prevention and control (Thatrimontrichai \& Apisarnthanarak, 2019).

Acknowledgments. The authors thank the office of International Affairs, Prince of Songkla University, Thailand, for the English editing.

\section{Source of Funding}

This work was supported only by the Faculty of Medicine, Prince of Songkla University, Thailand.

\section{Ethical approval}

The study was approved by the Ethical Committee Board of Faculty of Medicine, Prince of Songkla University (REC 58-26301-1).

\section{Conflicts of Interest}

On behalf of all authors, the corresponding author states that there is no conflict of interest. 


\section{REFERENCE}

Breurec, S., Bouchiat, C., Sire, J.M., Moquet, O., Bercion, R., Cisse, M.F., Glaser, P., Ndiaye, O., Ka, S., Salord, H., Seck, A., Sy, H.S., Michel, R. \& Garin, B. (2016). High third-generation cephalosporin resistant Enterobacteriaceae prevalence rate among neonatal infections in Dakar, Senegal. BMC Infectious Diseases 16: 587.

CLSI. (2018). Performance Standards for Antimicrobial Susceptibility Testing. CLSI supplement M10024 Nov 2018 [cited 201824 Nov]: Available from: https://clsi. org/standards/products/microbiology/ documents $/ \mathrm{m} 100 /$.

Cohen-Wolkowiez, M., Moran, C., Benjamin, D.K., Cotten, C.M., Clark, R.H., Benjamin, D.K., Jr. \& Smith, P.B. (2009). Early and late onset sepsis in late preterm infants. Pediatric Infectious Disease Journal 28: 1052-1056.

Goldstein, B., Giroir, B., Randolph, A. \& International Consensus Conference on Pediatric Sepsis. (2005). International pediatric sepsis consensus conference: definitions for sepsis and organ dysfunction in pediatrics. Pediatric Critical Care Medicine 6: 2-8.

Li, X., Xu, X., Yang, X., Luo, M., Liu, P., Su, K., Qing, Y., Chen, S., Qiu, J. \& Li, Y. (2017). Risk factors for infection and/or colonisation with extended-spectrum beta-lactamase-producing bacteria in the neonatal intensive care unit: a meta-analysis. International Journal of Antimicrobial Agents 50: 622-628.

Nivesvivat, T., Piyaraj, P., Thunyaharn, S., Watanaveeradej, V. \& Suwanpakdee, D. (2018). Clinical epidemiology, risk factors and treatment outcomes of extended-spectrum beta-lactamase producing Enterobacteriaceae bacteremia among children in a Tertiary Care Hospital, Bangkok, Thailand. BMC Research Notes 11: 624.

Simon, A. \& Tenenbaum, T. (2013). Surveillance of multidrug-resistant Gramnegative pathogens in high-risk neonates - does it make a difference? Pediatric Infectious Disease Journal 32: 407-409.
Thatrimontrichai, A. \& Apisarnthanarak, A. (2019). Active surveillance culture program in asymptomatic patients as a strategy to control multidrug-resistant gram-negative organisms: What should be considered? Journal of The Formosan Medical Association. In press.

Thatrimontrichai, A., Apisarnthanarak, A., Chanvitan, P., Janjindamai, W., Dissaneevate, S. \& Maneenil, G. (2013). Risk factors and outcomes of carbapenem-resistant Acinetobacter baumannii bacteremia in neonatal intensive care unit: a case-case-control study. Pediatric Infectious Disease Journal 32: 140-145.

Thatrimontrichai, A., Chanvitan, P., Janjindamai, W., Dissaneevate, S., Jefferies, A. \& Shah, V. (2014). Trends in neonatal sepsis in a neonatal intensive care unit in Thailand before and after construction of a new facility. Asian Biomedicine 8: 771-778.

Thatrimontrichai, A., Janjindamai, W., Dissaneevate, S. \& Maneenil, G. (2019a). Factors and burdens of central lineassociated bloodstream infections in a neonatal intensive care unit, Songkhla, Thailand. Southeast Asian Journal of Tropical Medicine and Public Health 50: 742-749.

Thatrimontrichai, A., Janjindamai, W., Dissaneevate, S., Maneenil, G. \& Kritsaneepaiboon, S. (2019b). Risk factors and outcomes of ventilatorassociated pneumonia from a neonatal intensive care unit, Thailand. Southeast Asian Journal of Tropical Medicine and Public Health 50: 537-545.

Thatrimontrichai, A., Premprat, N., Janjindamai, W., Dissaneevate, S. \& Maneenil, G. (2019c). Multidrug-resistant Gram-negative bacilli sepsis from a neonatal intensive care unit: a casecase-control study. Journal of Infection in Developing Countries 13: 603-611.

Thatrimontrichai, A., Techato, C., Dissaneevate, S., Janjindamai, W., Maneenil, G., Kritsaneepaiboon, S. \& Tanaanantarak, P. (2016). Risk factors and outcomes of carbapenem-resistant Acinetobacter baumannii ventilator- 
associated pneumonia in the neonate: a case-case-control study. Journal of Infection and Chemotherapy 22: 444449.

Tsai, M.H., Chu, S.M., Hsu, J.F., Lien, R., Huang, H.R., Chiang, M.C., Fu, R.H., Lee, C.W. \& Huang, Y.C. (2014). Risk factors and outcomes for multidrug-resistant Gram-negative bacteremia in the NICU. Pediatrics 133: e322-329.
Tsai, M.H., Lee, I.T., Chu, S.M., Lien, R., Huang, H.R., Chiang, M.C., Fu, R.H., Hsu, J.F. \& Huang, Y.C. (2016). Clinical and molecular characteristics of neonatal extended-spectrum beta-lactamaseproducing Gram-negative bacteremia: a 12-year case-control-control study of a referral center in Taiwan. PLoS One 11: $\mathrm{e} 0159744$. 